\title{
Análise Direcional de Erros Sistemáticos em Ortomosaico gerado por meio de ARP
}

\author{
Direcional analisys of systematic errors in orthomosaic generade by RPA
}

\author{
Dalva M. Castro Vitti ${ }^{1}$, Frederico Fábio Mauad², Ademir Marques Júnior ${ }^{3}$, \\ Leonardo Campos Inocêncio ${ }^{3}$, Maurício Roberto Veronez ${ }^{3}$
}

Recebido em abril de 2018.

Aprovado em novembro de 2018.

\begin{abstract}
RESUMO
A estatística circular é uma importante ferramenta para avaliar a tendência em mapeamentos a partir do imageamento com sensores embarcados. Este trabalho visou avaliar erros sistemáticos e em dois ortomosaicos obtidos do imageamento simultâneo com dois sensores embarcados no Hexator XFly 800. Foram gerados dois ortomosaicos pela técnica Structure from Motion. As discrepâncias foram obtidas pela comparação entre pontos homólogos observados nos ortomosaicos e coletados com GNSS RTK. Primeiramente, foram realizadas análises quanto a normalidade e aleatoriedade das discrepâncias observadas nos pontos de controle. De acordo com a função de Shapiro-Wilk as amostras não apresentaram distribuição normal e pelo teste de sequências foram consideradas aleatórias. Devido a não normalidade, foi realizada a análise direcional dos dados, obtendo-se assim as direções dos vetores resultantes de acordo com a função de von Mises. Para os ortomosaicos do sensor Canon ELPH 110 S e sequoia, os vetores direcionais resultantes ocorreram no azimute $47^{\circ}$ e $116^{\circ}$, com variâncias de $0,880 \mathrm{~m}$ e $0,810 \mathrm{~m}$, respectivamente, mostrando grande dispersão dos pontos e por consequência, concluindo pela não tendência de ambos ortomosaicos. O RMSE foi calculado para ambos ortomosaicos e comparados com o PECPCD, que conduziu ao enquadramento na Classe B da escala 1:2000.
\end{abstract}

PALAVRAS-CHAVE: Estatística circular. Exatidão cartográfica. Mapeamento digital.

\begin{abstract}
The circular statistic is an important tool to evaluate data tendency in mapping obtained from captured images with embedded sensors. This work aimed to evaluate systematic errors in two orthomosaics obtained from
\end{abstract}

\footnotetext{
${ }^{1}$ Programa de Pós-Graduação em Ciências da Engenharia Ambiental, Universidade de São Paulo Brasil. E-mail: dalva.vitti@fatec.sp.gov.br

${ }^{2}$ Programa de Pós-Graduação em Ciências da Engenharia Ambiental, Universidade de São Paulo. Brasil. E-mail: mauadffm@sc.usp.br

${ }^{3}$ Programa de Pós-Graduação em Computação Aplicada, Universidade do Vale do Rio dos Sinos. Brasil. E-mail: adejunior@edu.unisinos.br; lci@edu.unisinos.br; veronez@edu.unisinos.br
}

Rev. Bras. de Cartografia, vol. 70, Edição Especial "XXVII Congresso Brasileiro de 
simultaneous image capturing using two sensors embedded in the hex rotor XFly 800. The two orthomosaics were generated using the Structure from Motion technique, and the discrepancies were obtained comparing homologous points identified in the orthomosaic and points collected with the RTK GNSS. Firstly, the statistical analysis evaluated the sample regarding the distribution and randomness of the data. According the Shapiro-Wilk's test the sample did not present normal distribution, and besides that, we found that according the sequence test the data is random in both samples. Due to the not normal behavior of the samples, the directional analysis was performed, giving the resultant vector according to the Von Mises distribution. For the orthomosaics obtained from the Canon ELPH 110s and the Parrot Sequoia sensor, we found directional resultant vectors of $47^{\circ}$ and $116^{\circ}$, with variance of $0,880 \mathrm{~m}$ and $0,810 \mathrm{~m}$, respectively, showing representative dispersion of points, and consequently showing no tendency in both orthomosaics. The calculated RMSE for both orthomosaics was compared against the PEC-PCD standards, classifying these in the B class for the scale 1:2000.

KEYWORDS: Circular Statistics. Accuracy Cartography. Digital Mapping.

\section{Introdução}

A exatidão de produtos cartográficos baseia-se na análise de erros randômicos e sistemáticos comparados a um padrão estabelecido. A maioria dos padrões pressupõe a distribuição normal das discrepâncias observadas nos pontos de controle e assim, métodos estatísticos paramétricos são empregados para avaliação da qualidade posicional. Além disso, a amostra de pontos de controle deve ser independente e aleatória (SANTOS, 2015; ARIZA; ATKINSON, 2008).

Análise de dados direcionais tem sido aplicada em vários campos de pesquisa, como por exemplo, na migração de pássaros, na direção de ventos e escorregamentos glaciais (FISHER, 1993). Atkinson (2005) avalia a estatística circular como uma ferramenta poderosa para análise de dados eapaciais. Quando se trata de fotografias aéreas, é possível detectar padrões de orientação linear. Daí a importância de se dispor de ferramentas de análises circulares em 2D e esféricas em 3D para avaliar comportamentos sistemáticos, quanto à direção dos vetores de erros posicionais

Rev. Bras. de Cartografia, vol. 70, Edição Especial "XXVII Congresso Brasileiro de 
O mapeamento a partir do imageamento com sensores embarcados em ARP (Aeronave Remotamente Pilotada) fundamenta-se em conceitos da visão computacional de modelagem baseada no objeto (DEBEVEC et al., 1996; REMONDINO et al., 2011) em que o algoritmo Structure from Motion - SfM reconstrói a posição das câmeras a partir da detecção e descrição de pontos de interesse num conjunto de imagens resultando numa nuvem esparsa de pontos (FONSTAD et al., 2013, SNAVELY et al., 2008, SZELISKI, 2010).

Para SfM é indiferente se a coleta de imagens foi realizada com câmera métrica ou não métrica. O mais importante é garantir que a separação angular entre as imagens não supere 25 a 30 graus, obtido com uma sobreposição de $60 \%$ a $80 \%$ para imagens capturadas de um único local, e assegurar que cada objeto fotografado tenha uma textura única para o bom desempenho dos algoritmos de deteç̧ão e descrição dos elementos (MOREELS; PERONA, 2007; CORSI; SLAPŠAK; VERMEULEN, 2013).

As coordenadas fornecidas pelo GNSS da aeronave e a composição RGB do ponto de tomada da imagem no terreno, capturada pela câmera e atitude da aeronave durante a coleta de imagens descrita pela IMU com as rotações em torno dos eixos x, y e z (ômega, phi e Kapa), compõem o log de voo, o qual será utilizado pelo SfM para cumprir as etapas até a geração da nuvem de pontos esparsa.

A detecção de elementos em SfM corresponde a identificação de pontos de interesse (PI ou keypoints) que possuem as seguintes características: textura inconfundível em relação a seus vizinhos, alta repetibilidade, invariância na iluminação, ruídos na imagem e transformações geométricas básicas como escala, translação, rotação e corte (SNAVELY; SEITZ; SZELISKI, 2008; SZELISKI, 2010). O algoritmo mais utilizado nesta etapa é o SIFT (Scale Invariant Feature Transform), que detecta uma região comum em várias fotografias (LOWE, 1999; BROWN; LOWE, 2002; MOREELS; PERONA, 2007).

$\mathrm{Na}$ descrição dos elementos em SfM, o algoritmo busca localizar os PIs $2 \mathrm{D}$ que representam o mesmo ponto físico $3 \mathrm{D}$, em que cada conjunto de pontos 
codificados com a mesma cor corresponda a projeção do mesmo ponto 3D. O descritor de feições calcula um vetor do elemento com as características locais para descrever uma correção local de pixels em torno de cada PI. Assim como os PIs, esses vetores devem ser invariantes em escala (CORSI; SLAPŠAK; VERMEULEN, 2013).

$\mathrm{Na}$ etapa do descritor de correspondência, todos os vetores descritos na fase anterior são combinados em pares em diferentes imagens através da junção de elementos semelhantes, onde é calculada a distância entre os elementos, por exemplo, a distância euclidiana. A dimensão dos vetores tem impacto direto no tempo de processamento. Este processo é realizado em todos os pares de imagens, verificando se a suposição das localizações das correspondências cumpre a restrição da geometria Epipolar, ou seja, a geometria intrínseca em dois pontos de vistas. A geometria Epipolar independe da estrutura da cena e dependente apenas dos parâmetros internos da câmera e da posição relativa (SZELISKI, 2010).

$\mathrm{Na}$ etapa de triangulação, a SfM calcula a localização dos pontos num sistema de referência local, criando uma nuvem esparsa de pontos 3D. A triangulação na imagem requer que sejam conhecidas as orientações interior e exterior da imagem, que é conseguida após a combinação de todas as orientações relativas, em pares, na forma de matriz fundamental. Uma matriz fundamental é a representação algébrica da geometria epipolar 3 x 3 que relaciona os pontos correspondentes em imagens estéreo, Figura 1.

Assim, pelo teorema da reconstrução projetiva, um conjunto de pontos em duas vistas é definido pela matriz fundamental. Então, a geometria da cena 3D e a matriz de projeção das imagens podem ser reconstruídas a partir da correspondência de quaisquer duas reconstruções projetivamente equivalentes (SZELISKI, 2010).

Figura 1 - Triangulação 3D e geometria epipolar na identificação do ponto P em SfM. 

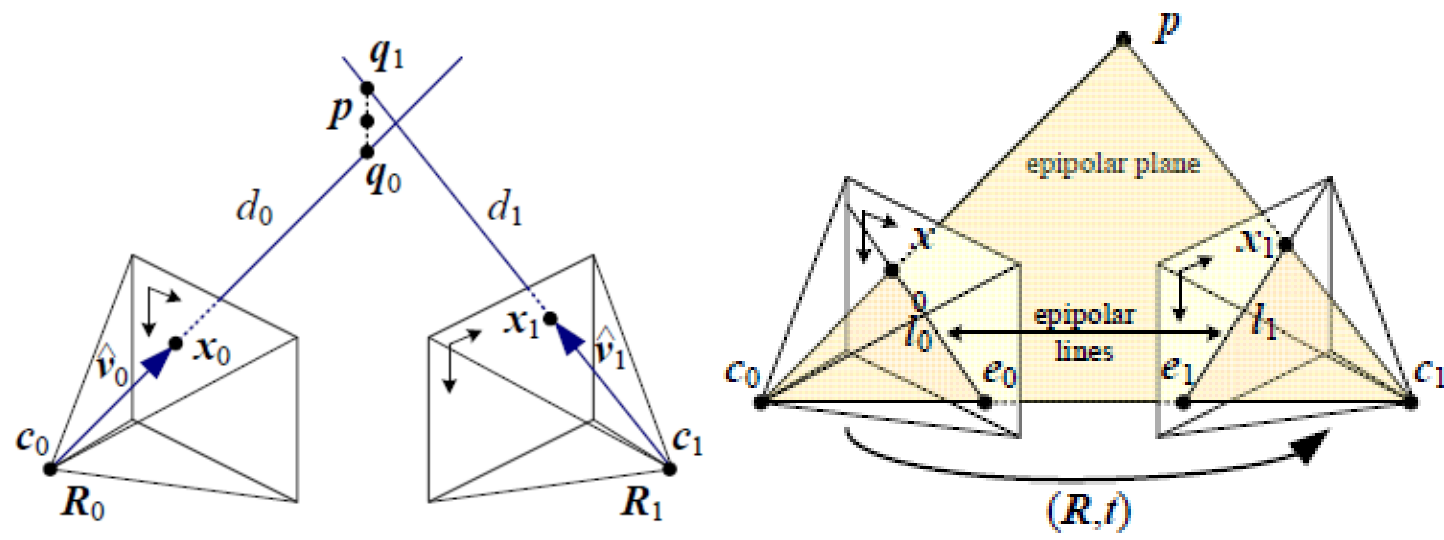

Fonte: Szeliski (2010).

Após triangulação, os pares de imagem orientados na matriz fundamental são combinados para formar o bloco completo de imagens e gerar a estrutura da cena. Dessa forma, tem-se um modelo sub-otimizado porque nem todas as imagens foram sobrepostas e há discrepâncias na estrutura devido a alguns erros algébricos ocorridos na fase de medição. Para superar esse problema, a maioria dos algoritmos SfM realiza o ajustamento de blocos, Bundle Block Adjustment (TRIGGS et al., 2000; NI; STEEDLY; JEONG et al., 2012), o qual otimiza iterativamente a estrutura 3D e as matrizes de projeção de todas as imagens, simultaneamente, executando uma minimização não linear da medida real. Isto significa dizer que, numa abordagem SfM, a estrutura da cena e as matrizes da câmera são recuperadas sem o conhecimento da posição da câmera ou orientação interior (HARTLEY; ZISSERMAN, 2003; SZELISKI, 2010).

O georreferenciamento num sistema absoluto é feito com inserção de pontos de apoio (GARCÍA, 2002). Coveney e Roberts (2017) conseguiram exatidão suficiente a partir de 5 pontos de controle rastreados com GNSS RTK para modelagem do modelo digital de elevação - MDE.

A exatidão de ortomosaico gerado a partir do mapeamento com RPAS tem sido avaliada em termos do RMSE (Root Mean Square Error) comparando-se a posição de pontos ou nuvem de pontos observados na 
imagem e coletados no campo por uma técnica mais precisa (JAUD et al., 2016; HARWIN; LUCIER, 2012).

No Brasil, a exatidão de produtos cartográficos adota como referência o padrão de erro cartográfico (PEC) que pode ocorrer em função da escala, definido pelo Decreto-Lei n. ${ }^{\circ} 89.817$ de 1984 (BRASIL, 1984). Para produtos cartográficos digitais, combina-se este decreto com as Especificações Técnicas de Aquisição de Dados Geoespaciais Vetoriais (ET-ADGV). No entanto, para aplicação desta norma, pressupõe-se que a amostra apresente distribuição normal de probabilidades.

ASPRS (2015), recomenda que métodos robustos sejam estudados para análise de exatidão de produtos cartográficos, uma vez que, a maioria dos padrões de exatidão tem a normalidade da amostra como pressuposto, e isso nem sempre ocorre.

Santos et al (2016), comentam que a análise de tendência dos dados é importante para evitar o comprometimento da qualidade do produto cartográfico e para evitar análises e decisões equivocadas quanto a realidade do campo. Geralmente, os erros sistemáticos decorrem da falta de controle nas várias etapas da produção cartográfica, como erros de transformação de sistemas de referenciais geodésicos, erros de projeção, confusões com metadados, uso de equipamentos não retificados.

Este trabalho visou determinar erros sistemáticos $2 \mathrm{D}$ em dois ortomosaicos gerados a partir do imageamento simultâneo com dois sensores embarcados em um hexarotor por meio da estatística circular.

\section{Análise de exatidão de produtos cartográficos}

\subsection{Amostragem de pontos de controle}

De acordo com Merchant (1982), 20 pontos é o número mínimo de pontos para verificação da distribuição de probabilidade da amostra que pode conduzir a aceitação ou rejeição da hipótese quanto a normalidade. No 
entanto, segundo Mann (2006), 30 pontos são indicados para o caso da rejeição da hipótese nula e aplicação de métodos não paramétricos de análise estatística.

Os pontos de controle para verificação da exatidão cartográfica são obtidos por métodos mais precisos, seja com coletas de campo (GNSS) ou identificados em uma base cartográfica de maior precisão. Esses pontos são comparados com seus homólogos extraídos do produto cartográfico em análise, e dessa forma, obtendo-se os valores das discrepâncias.

Domingo (2000) recomenda que após o cálculo das discrepâncias, façase a inspeção de pontos anômalos (outliers) antes de se seguir com os testes estatísticos para verificação de exatidão de produtos cartográficos. Essa inspeção pode ser feita por análise de gráficos box-plot (SANTOS et al., 2016) ou estabelecendo-se um limite em função do desvio padrão amostral (i.e, 3 vezes o desvio padrão).

\subsection{Teste de normalidade das discrepâncias}

O teste de normalidade deve ser aplicado a uma amostra para que o analista tome a decisão correta quanto aos testes a serem aplicados na avaliação da exatidão de produtos cartográficos. Se a amostra apresentar distribuição normal de probabilidades, os testes paramétricos e os estimadores, média, desvio padrão, representam satisfatoriamente a qualidade da população de uma base de dados espaciais. Porém, quando as discrepâncias apresentam distribuição livre (não normal), testes robustos devem ser aplicados para avaliar a qualidade do produto cartográfico (ATKINSON, 2005; SANTOS et al., 2016).

O teste de Shapiro-Wilk (1965) foi utilizado na avaliação da normalidade das discrepâncias observadas em produtos cartográficos (SANTOS et al. 2016). Este teste apresenta melhor desempenho na análise de dados de distribuição livre quando comparado com Kolmogorov-Smirnov, Qui quadrado e teste t (TORMAN; COSTER; RIBOLDI, 2012). 
O teste de Shapiro-Wilk (1965) consiste na determinação do valor W, dado pela Equação 1:

$$
W=\frac{b^{2}}{\sum_{i=1}^{n}\left(x_{i}-\bar{x}\right)^{2}}
$$

Sendo b dado por:

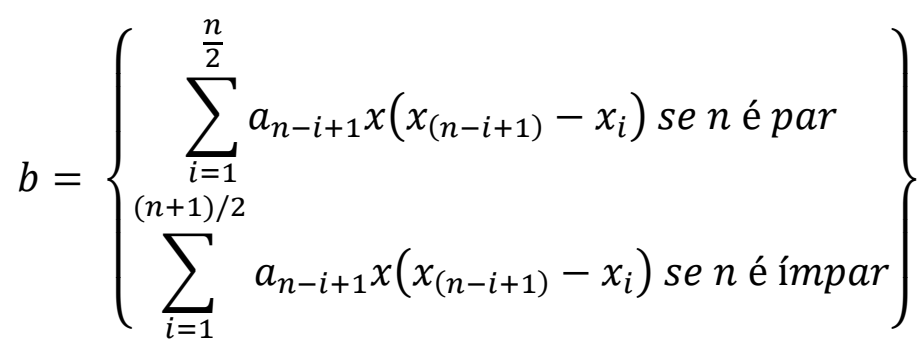

em que a n-(i+1) são constantes geradas pela média, variância e covariância das estatísticas de ordem de uma amostra de tamanho n numa distribuição normal.

No teste de Shapiro-Wilk, são realizadas as seguintes etapas:

a) Formula-se a hipótese de que os dados amostrais provêm de uma distribuição normal, sendo:

$$
\begin{gathered}
\mathrm{H}_{0}=\text { amostra provém de uma população normal } \\
\mathrm{H}_{1}=\text { amostra não provém de uma distribuição normal }
\end{gathered}
$$

b) Estabelece-se o nível de significância, geralmente, $a=0,05$;

c) Calcula-se a estatística de teste, o ordenamento dos valores amostrais, cálculo de b e cálculo de W. Então, toma-se uma decisão. Rejeita-se $\mathrm{H}_{0}$ se Wcalc $>$ Wtab, ao nível de significância de a.

A decisão também pode ser baseada no p-valor, que representa um índice de força para a natureza que está sendo testada, com base em valores 
mais extremos que a estatística de amostra de teste. Sempre que o nível de significância for maior que o p-valor, pode-se rejeitar a hipótese nula.

Os padrões de verificação da exatidão presumem que pelo menos $90 \%$ dos dados enquadrem-se num intervalo da média mais duas vezes o desvio padrão. Essa condição só é conseguida quando a hipótese nula é atendida. Quando a hipótese alternativa é confirmada, ou seja, as discrepâncias não apresentam uma distribuição normal, testes estatísticos não paramétricos, apesar de menos seguros que testes paramétricos, podem caracterizar melhor a condição dos dados.

\subsection{Teste de aleatoriedade das discrepâncias}

A aleatoriedade das discrepâncias foi verificada por meio do teste de sequências ou Runs Test (BRADLEY, 1968 apud MANN, 2006). Atkinson (2005) apresentou este teste em sua proposta para verificação da exatidão horizontal por ser de fácil aplicação.

O teste de sequências representa um conjunto com um ou mais arranjos consecutivos do mesmo resultado, em uma série de ocorrências, na qual existam somente dois resultados (MANN, 2006). No caso da análise de

aleatoriedade das discrepâncias, as hipóteses prévias definidas são as seguintes:

$\mathrm{H}_{0}$ : discrepâncias são aleatoriamente distribuídas

$\mathrm{H}_{1}$ : discrepâncias não são aleatoriamente distribuídas

Para realização do teste, determina-se a mediana e as hipóteses prévias são elaboradas da seguinte forma:

$$
\begin{aligned}
& \mathrm{H}_{0}: \text { discrepância } \geq \text { mediana } \\
& \mathrm{H}_{1}: \text { discrepância }<\text { mediana }
\end{aligned}
$$


A resposta ao teste será do tipo sim ou não, ou + ou -, ou ainda, 0 ou 1. Então, as discrepâncias são separadas em dois grupos: $\mathrm{n}_{1}$ para as positivas a $\mathrm{H}_{0}$ e $\mathrm{n}_{2}$ para as respostas que contrariam $\mathrm{H}_{0}$. Em seguida, conta-se o número de arranjos designado por $\mathrm{R}$ como sendo o evento de cada sequência de sim ou não (por ex., SSNNNSNSS, $R=5$ ). Portanto, $R$ representa uma estatística com sua própria distribuição de amostragem. Os valores críticos de $\mathrm{R}$ são determinados por meio de uma tabela de Valores Críticos para um teste bicaudal igual a 0,05 ( $\mathrm{n}_{1}$ e $\mathrm{n}_{2}$ menores que 15), senão, calcula-se o valor de $\mathrm{Z}$ em função da média de $\mathrm{R}\left(\mu_{\mathrm{R}}\right)$ e do desvio padrão de $\mathrm{R}\left(\sigma_{\mathrm{R}}\right)$ (Equações 2 a 4).

$$
\begin{gathered}
Z=\frac{R-\mu_{R}}{\sigma_{R}} \\
\mu_{R}=\frac{2 \cdot n_{1} \cdot n_{2}}{n_{1}+n_{2}}+1 \\
\sigma_{R}=\sqrt{\frac{2 \cdot n_{1} \cdot n_{2}\left(2 \cdot n_{1} \cdot n_{2}-n_{1}-n_{2}\right)}{\left(n_{1}+n_{2}\right)^{2}\left(n_{1}+n_{2}-1\right)}}
\end{gathered}
$$

Conhecidos o valor Z e o grau de confiança $(0,01<\mathrm{a}<0,05)$, busca-se na tabela da distribuição normal padronizada o valor de $\mathrm{Z}_{\text {crítico. Então, toma- }}$ se uma decisão aceitando ou não a hipótese nula.

$\mathrm{O}$ teste da mediana apresenta como resultado que pelo menos $50 \%$ dos pontos testados enquadram-se na hipótese nula com grau de confiança estabelecido, atestando a aleatoriedade da amostra.

\subsection{Erro direcional}


Para a verificação de erros sistemáticos $2 \mathrm{D}$ foi adotada a distribuição de von Mises que opera de forma semelhante a distribuição normal, com parâmetros que indicam suas características: direção $(\theta)$, média $(\mu)$ e parâmetro de concentração (k) (JAMMALAMADAKA; SEN-GUPTA, 2001; JUPP; MARDIA, 1989). A variável aleatória circular "Y" tem distribuição de von Mises, com parâmetros $\mu \mathrm{e} \mathrm{k}$, se sua função densidade de probabilidade for dada pela Equação 6. Foi atribuído ao fator k valor igual a 0,5.

$$
f(y, \mu, k)=\frac{1}{2 \pi I_{0}} \exp [k \cos (y-\mu)]
$$

para $0 \leq y \leq 2 \pi ; 0 \leq \mu \leq 2 \pi ;$ e $k>0$

onde $\mathrm{I}_{0}(\mathrm{k})$ é a função de Bessel modificada de $1^{\circ}$ tipo e ordem zero, que é dada pela série (Equação 7):

$$
I_{0}(k)=\sum_{r=0}^{\infty} \frac{1}{r !^{2}}\left(\frac{1}{2} k\right)^{2}
$$

Dada uma série de vetores unitários, sua direção predominante é representada pela resultante das discrepâncias das coordenadas X e Y, Equações 8 e 9, e o ponto final do vetor unitário, cuja direção é formada pelo ângulo $(\theta)$ em relação aos eixos x e y (JAMMALAMADAKA; SEN-GUPTA, 2001).

$$
\begin{aligned}
& X_{i}=\cos \theta \\
& Y_{i}=\operatorname{sen} \theta
\end{aligned}
$$

Considerando C a soma das componentes Xi, Equação 10 e S, a soma das componentes Yi, Equação 11, tem-se a direção predominante do erro sistemático dado pelo ângulo $(\theta)$, Equação 12. E a resultante $\mathrm{R}$ é dada pela Equação 13: 


$$
\begin{gathered}
C=\sum_{i=1}^{n} \cos \theta \\
S=\sum_{i=1}^{n} \operatorname{sen} \theta \\
\theta=\operatorname{atan}\left(\frac{S}{C}\right) \\
\mathrm{R}^{2}=\mathrm{S}^{2}+\mathrm{C}^{2}
\end{gathered}
$$

Com $\mathrm{R}$ variando entre [0,1]. Quanto mais próximo de 0 é $\mathrm{R}$, maior é a dispersão dos dados.

A média circular de $\theta_{1}, \theta_{2}, \theta_{3}, \ldots, \theta$ n é definida como sendo o ângulo médio correspondente ao vetor resultante, que deve satisfazer as seguintes condições (Equações 14 e 15):

$$
\begin{aligned}
& \cos (\bar{\mu})=\frac{C}{R} \\
& \operatorname{sen}(\bar{\mu})=\frac{S}{R}
\end{aligned}
$$

se:

$$
\bar{\mu}=\left\{\begin{array}{c}
\operatorname{atan}\left(\frac{S}{C}\right), \text { se } S \geq 0 \text { e } C>0 \\
\operatorname{atan}\left(\frac{S}{C}\right)+\pi, \quad \text { se } C<0 \\
e \\
\operatorname{atan}\left(\frac{S}{C}\right)+2 \pi, \text { se } S<0 \text { e } C>0
\end{array}\right\}
$$

A magnitude da resultante $\mathrm{R}$ fornece uma informação sobre o grau de concentração dos ângulos observados. Assim, quanto maior a dispersão da amostra, menor a resultante (OTIENO, 2002). Então, para se comparar duas resultantes é mais comum usar o comprimento médio, $\bar{R}$, dado em função das componentes médias de C e S (Equações 16, 17 e 18), no qual tem a vantagem de variar no intervalo $[0,1]$. 


$$
\begin{gathered}
\bar{C}=\frac{N_{r}}{n}=\frac{1}{n} \sum_{i=1}^{n} \cos \theta \\
\bar{S}=\frac{E_{r}}{n}=\frac{1}{n} \sum_{i=1}^{n} \operatorname{sen} \theta \\
\bar{R}=\frac{R}{n}=\sqrt{\overline{C^{2}}+\overline{S^{2}}}
\end{gathered}
$$

A variância mínima ocorre quando $\mathrm{V}=0$ e corresponde a todas as observações terem a mesma direção. Assim, a média direcional pode ser utilizada para representar a direção do conjunto de vetores. O maior limite possível de variação ocorre para dados uniformemente distribuídos ao redor do círculo, e corresponde a $\mathrm{V}=1$. Neste caso, a média direcional não representa a tendência da direção dos dados cartográficos (SANTOS et al., 2016). A variância e o desvio padrão são dados pelas Equações 19 e 20 (OTIENO, 2002):

$$
\begin{gathered}
V=1-\bar{R} \\
\sigma=\sqrt{-2 \cdot \ln \bar{R}}
\end{gathered}
$$

\subsubsection{Teste de significância da média direcional}

O teste de significância da estatística circular ou teste de Hayleigh baseia-se nas hipóteses prévias formuladas em relação a média direcional:

Ho: a amostra possui média direcional

H1: a amostra não possui média direcional

A decisão é tomada sobre o valor de $\mathrm{Z}$ calculado em função do tamanho da amostra e da resultante direcional (Equação 21). Então, $\mathrm{Z}_{\text {calculado é }}$ comparado com valor de $\mathrm{Z}_{\text {tabelado }}$ do teste de Hayleigh.

$$
\text { Zcalc }=\mathrm{n} \cdot \mathrm{R}^{2}
$$




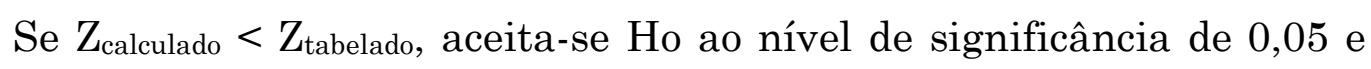
conclui-se que a amostra possui média direcional. Do contrário, rejeita-se Ho.

2.3.2 Teste de uniformidade da estatística circular

Os azimutes formados pelas discrepâncias $\mathrm{E}$ e $\mathrm{N}$ apresentam distribuição uniforme, caso suas posições no círculo geográfico forem uniformes com base nas seguintes hipóteses:

Ho: a distribuição dos azimutes é uniforme

$\mathrm{H}_{1}$ : a distribuição dos azimutes não é uniforme

O círculo geográfico é dividido em 8 partes iguais, de $45^{\circ}$ em $45^{\circ}$, onde são considerados azimutes Norte todos que se localizarem entre os azimutes $337,5^{\circ}$ e $22,5^{\circ}$, nordeste entre $22,5^{\circ}$ e $67,5^{\circ}$ e assim por diante, o que completa 8 grupos, como apresentado na Tabela 1. Sendo o tamanho da amostra maior que 30, calcula-se o valor de Qui quadrado para a amostra com base na frequência de ocorrência dos azimutes em cada categoria (fi), em relação a frequência absoluta ( fi) dados pelas Equações 22 e 23.

Tabela 1 - Categorias de azimutes para teste de uniformidade da estatística circular.

\begin{tabular}{lcc}
\hline Categoria de Azimutes & Localização & Frequência (fi) \\
\hline $337,5^{\circ}-22,5^{\circ}$ & Norte & $\mathrm{n} 1$ \\
\hline $22,5^{\circ}-67,5^{\circ}$ & Nordeste & $\mathrm{n} 2$ \\
\hline $67,5^{\circ}-112,5^{\circ}$ & Leste & $\mathrm{n} 3$ \\
\hline $112,5^{\circ}-157,5^{\circ}$ & Sudeste & $\mathrm{n} 4$ \\
\hline $157,5^{\circ}-202,5^{\circ}$ & Sul & $\mathrm{n} 5$ \\
\hline $202,5^{\circ}-247,5^{\circ}$ & Sudoeste & $\mathrm{n} 6$ \\
\hline $247,5^{\circ}-292,5^{\circ}$ & Oeste & $\mathrm{n} 7$ \\
\hline $292,5^{\circ}-337,5^{\circ}$ & Noroeste & $\mathrm{n} 8$ \\
\hline & Fonte: JAMMALAMADAKA; SEN-GUPTA (2001).
\end{tabular}

Rev. Bras. de Cartografia, vol. 70, Edição Especial "XXVII Congresso Brasileiro de 


$$
\begin{gathered}
\chi_{c a l}^{2}=\sum \frac{\left(f i-\widehat{f}_{l}\right)^{2}}{f i} \\
\widehat{f}_{l}=\frac{n}{8}
\end{gathered}
$$

Então, confronta-se $\chi_{c a l}^{2}$ com $\chi_{t a b}^{2}$. Se $\chi_{c a l}^{2}>\chi_{t a b}^{2}$ rejeita-se a hipótese nula de que a distribuição dos azimutes é uniforme para n-1 graus de liberdade e nível de confiança de 0,05.

\subsection{Avaliação da Exatidão do produto cartográfico}

O RMSE, Equação 24, indica o erro médio cometido em cada posição, dado pela raiz quadrada da razão entre o quadrado das discrepâncias e o tamanho da amostra. O RMSE independe do tipo de função da distribuição amostral. Por isso, é bastante utilizado para representar a exatidão de produtos cartográficos.

$$
R M S E_{c}=\sqrt{\frac{1}{n} \sum_{i=1}^{i=n} e_{i}^{2}}
$$

Para avaliação da exatidão posicional de produtos cartográficos digitais, de acordo com o Padrão de Exatidão Cartográfica brasileiro, o RMSE é comparado com valores limites do erro padrão (EP) e se este for menor, fazse o enquadramento em função da escala e classe, como mostrado na Tabela 2 .

Tabela 2 - Padrão de exatidão cartográfica planimétrico de produtos cartográficos digitais.

\begin{tabular}{lcccc}
\hline Classe & PEC Planimétrico & EP planimétrico & PEC Altimétrico & EP altimétrico \\
\hline $\mathrm{A}$ & $0,5 \mathrm{~mm} \times$ escala & $0,3 \mathrm{~mm} \times$ escala & $1 / 2$ Equid. CN & $1 / 3$ Equid. CN \\
$\mathrm{B}$ & $0,8 \mathrm{~mm} \times$ escala & $0,5 \mathrm{~mm} \times$ escala & $3 / 5$ Equid. CN & $2 / 5$ Equid. CN \\
$\mathrm{C}$ & $1,0 \mathrm{~mm}$ x escala & $0,6 \mathrm{~mm} \times$ escala & $3 / 4$ Equid. CN & $3 / 8$ Equid. CN \\
\hline
\end{tabular}

${ }^{1}$ Equid. CN - Equidistância entre curvas de nível.

Fonte: BRASIL (1984).

\section{Experimento Prático}


3.1 Coleta de dados e geração de ortomosaicos

Primeiramente foi realizada a coleta simultânea de imagens sobre o campus da Universidade do Vale do Rio dos Sinos, Figura 2, com os sensores Canon ELPH 110S e Parrot Sequoia embarcados no hexarotor ST 800 da XFly, Figura 3, a uma altitude média de $120 \mathrm{~m}$. Na Tabela 3, são apresentadas as principais características dos sensores embarcados e das imagens obtidas.

Figura 2 - Localização da área mapeada destacada no retângulo.

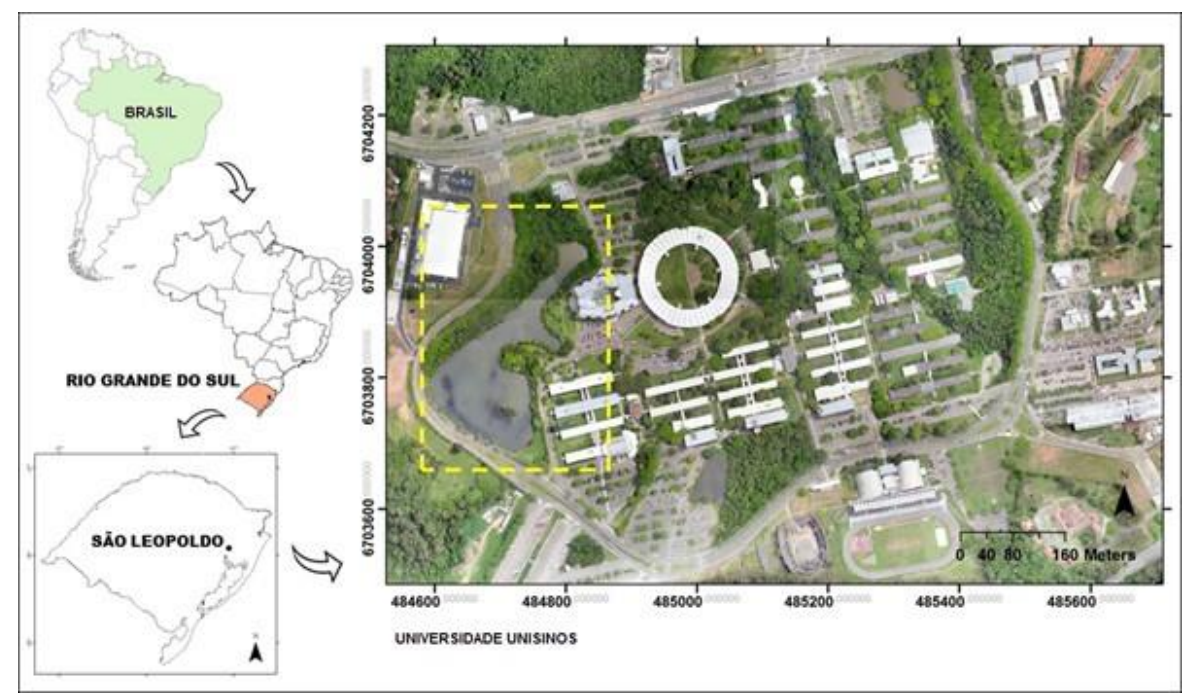

Fonte: Elaborada pelos autores.

Figura 3 - Hexator Xfly ST 800 e sensores embarcados.
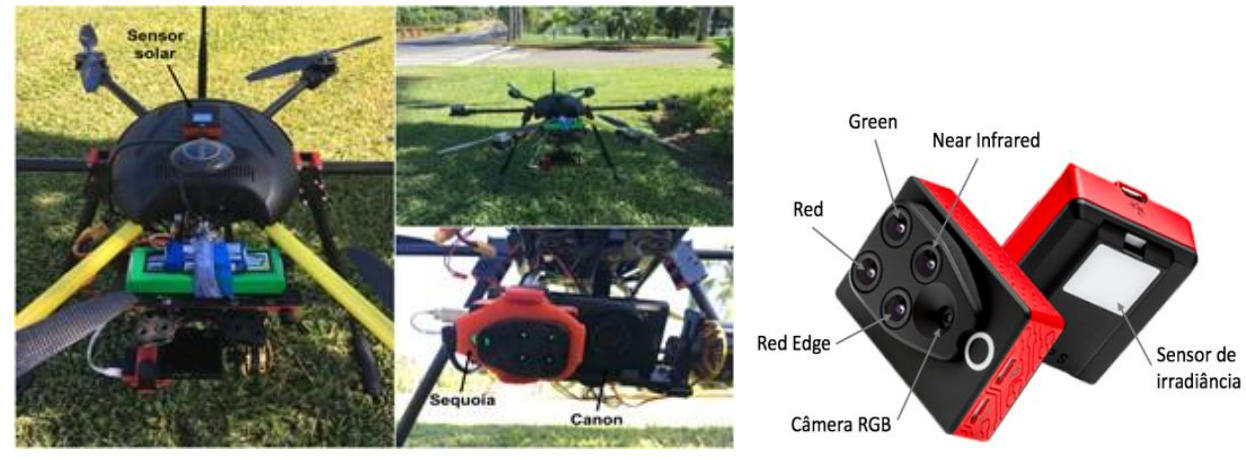

Fonte: Elaborada pelos autores.

Tabela 3 - Características básicas da câmera RGB CANON ELPH 110HS e do sensor

Parrot Sequoia e das imagens coletadas.

Rev. Bras. de Cartografia, vol. 70, Edição Especial "XXVII Congresso Brasileiro de 


\begin{tabular}{|c|c|c|c|c|c|c|c|}
\hline $\begin{array}{l}\text { Câmera/ } \\
\text { Sensor }\end{array}$ & Resolução & $\begin{array}{c}\text { Faixa } \\
\text { espectral }\end{array}$ & $\begin{array}{c}\text { Distância } \\
\text { focal } \\
(\mathrm{mm})\end{array}$ & $\begin{array}{l}\text { Dimensões } \\
\text { do sensor }\end{array}$ & $\begin{array}{c}\text { Tamanho } \\
\text { da } \\
\text { imagem } \\
\text { no pixel } \\
(\mathrm{mm})\end{array}$ & $\begin{array}{c}\text { Tamanho } \\
\text { do pixel } \\
\text { (cm) }\end{array}$ & $\begin{array}{l}\text { GSD } \\
(\mathrm{cm})\end{array}$ \\
\hline $\begin{array}{l}\text { CANON } \\
\text { ELPH } \\
110 H S\end{array}$ & $16,1 \mathrm{MP}$ & RGB & 4,4 & $\begin{array}{c}6,17 \mathrm{x} \\
4,63\end{array}$ & ---- & 5,00 & 15,00 \\
\hline $\begin{array}{l}\text { Parrot } \\
\text { Sequoia } \\
4.0\end{array}$ & $16 \mathrm{MP}$ & $\begin{array}{l}\text { RGB } \\
\text { Red } \\
\text { Edge } \\
\text { NIR }\end{array}$ & ------ & ------ & $\begin{array}{c}1280 \mathrm{x} \\
960\end{array}$ & 14,52 & 14,52 \\
\hline
\end{tabular}

Fonte: elaborada pelos autores

Em seguida, 38 pontos de controle foram coletados com GNSS RTK em feições identificadas na sinalização horizontal da via no entorno do lago, sendo que destes, 7 pontos foram utilizados para georreferenciamento do modelo digital de elevação (MDE) e 31 pontos foram utilizados para checagem da qualidade cartográfica do ortomosaico.

As imagens foram processadas no software PIX4D que emprega a técnica Struture from Motion na reconstrução do modelo digital do terreno. Desse processamento, resultou dois ortomosaicos com GSD (ground sample distance) de $15 \mathrm{~cm}$, Figura 4 e Figura 5.

Em seguida, foram calculadas as discrepâncias entre os pontos de controle coletados em campo e seus homólogos extraídos do ortomosaico, conforme item 2.1. A inspeção de outliers, foi feita comparando-se a maior discrepância com 3 x o desvio padrão da amostra.

Figura 4 - Ortomosaico obtido com imagens da câmera Canon ELPH 110 S 


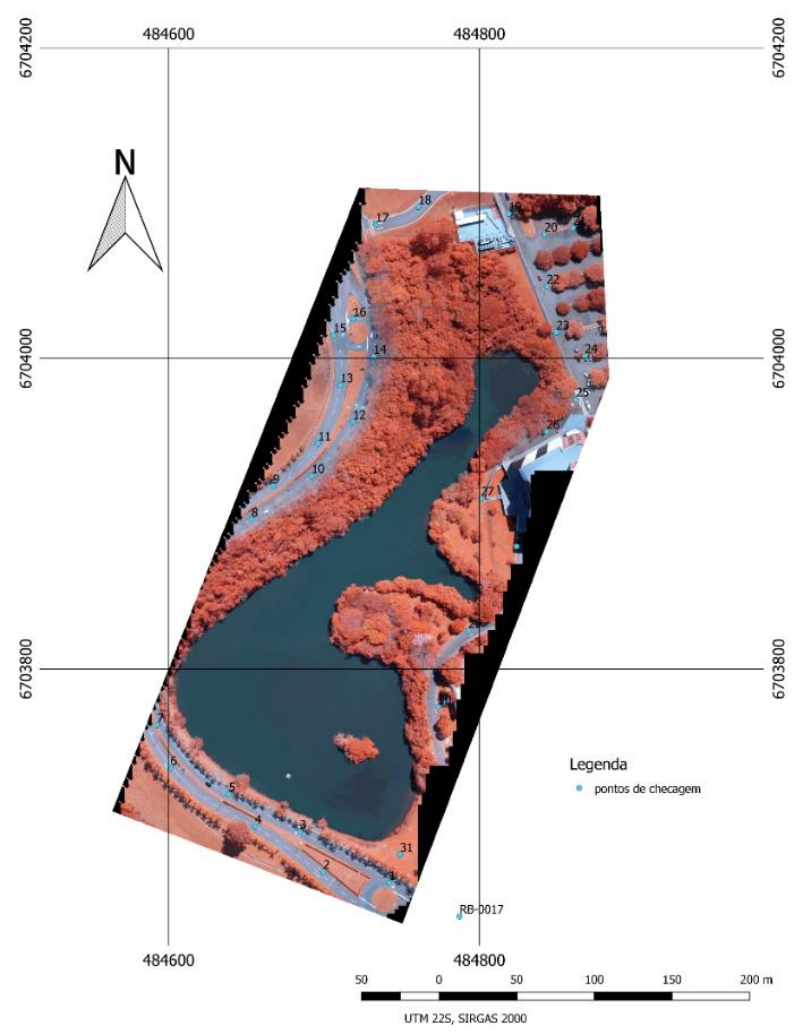

Fonte: Elaborada pelos autores.

Figura 5-Ortomosaico gerado com imagens do sensor Sequoia com 31 pontos de checagem.

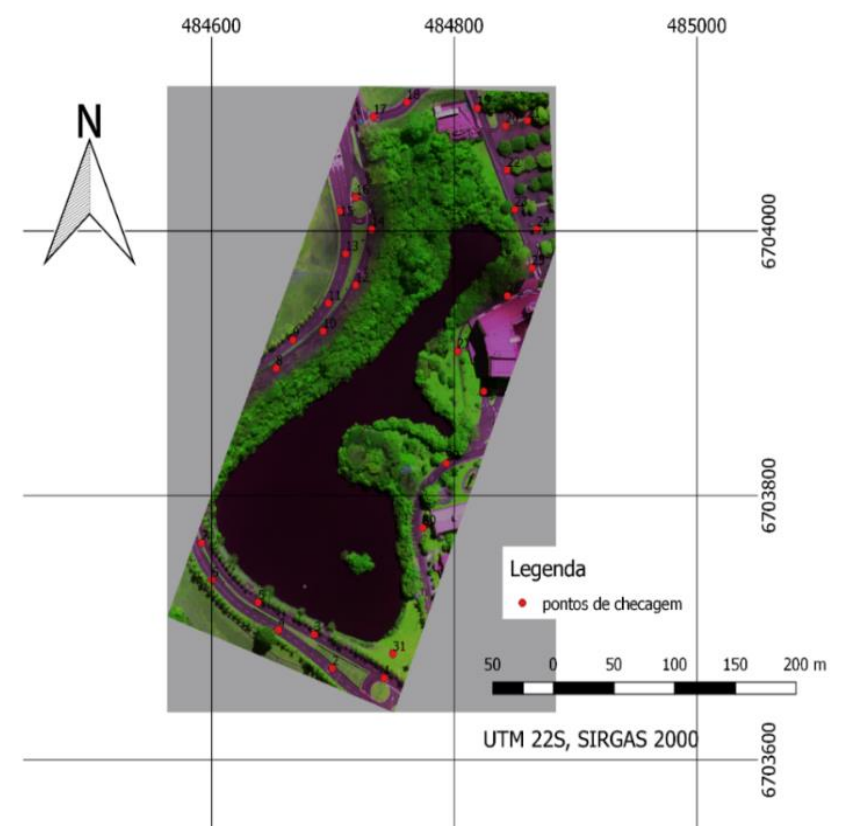

Fonte: Elaborada pelos autores.

3.2 Teste de normalidade das discrepâncias E N

Rev. Bras. de Cartografia, vol. 70, Edição Especial "XXVII Congresso Brasileiro de Cartografia”, 2018. pp. 1566 - 1594. 
A normalidade das discrepâncias em E N observadas nos ortomosaicos gerados com imagens dos sensores Canon ELPH 110S e Sequoia foi verificada por meio da análise do histograma comparado com a função de distribuição de probabilidades de Shapiro-Wilk (1965), Figura 6.

Figura 6 - Teste de normalidade das discrepâncias EN dos ortomosaicos gerados com as imagens coletadas através do sensor Canon ELPH 110S + SfM em (a) e Sequoia em (b).

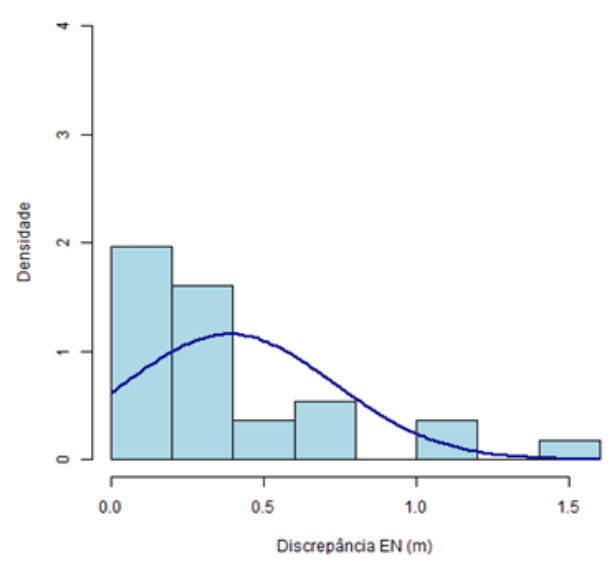

(a)

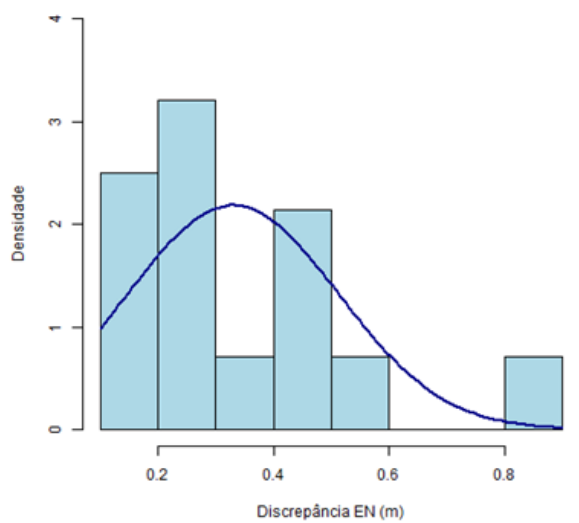

(b)

Fonte: Elaborada pelos autores.

O p-valor do teste de Shapiro-Wilk para as discrepâncias E e N observadas no ortomosaico, com origem nas imagens da câmera Canon ELPH 110S + SfM, foi de 0,00 e 0,01. Como p-valor, foi menor que 0,05, os resultados conduzem a não aceitar a hipótese nula ao nível de confiança de 95\%, ou seja, as discrepâncias não apresentaram distribuição normal.

Diante desse resultado, foi realizado um novo teste aplicando-se o Teorema do Limite Central (TLC). Foram calculadas as médias, agrupandose as discrepâncias 3 a 3, e realizado um novo teste de normalidade. Nessa iteração, p-valor foi de 0,001 para EN. Novamente, confirmou-se que a hipótese nula não deve ser aceita ao nível de significância de 0,05 e com 27 graus de liberdade.

As discrepâncias EN dos produtos do sensor Sequoia também não apresentaram distribuição normal de acordo com o teste de Shapiro-Wilk. De 
forma análoga ao executado com as discrepâncias da câmera Canon ELPH $110 \mathrm{~S}$, foi aplicado o TLC, agrupando-se os dados 3 a 3. Os resultados conduziram a não normalidade, confirmando que a hipótese nula não foi aceita ao nível de significância de $95 \%$ e com 27 graus de liberdade.

Como a análise de normalidade das discrepâncias pelo teste de Shapiro-Wilk conduziram a não aceitação da hipótese nula, então, partiu-se para a análise de exatidão por métodos não paramétricos. Então, foi aplicado o teste para verificação da aleatoriedade dos dados e, no caso, da análise de tendência nas direções EN, aplicou-se o teste direcional. E, para análise da exatidão posicional, o RMSE foi confrontado com o PEC-PCD.

\subsection{Análise da aleatoriedade dos dados}

A aleatoriedade das discrepâncias EN foi verificada pelo teste de sequências, como apresentado no item 2.3. Determinou-se a mediana das amostras EN, e confrontou-se com as hipóteses prévias.

$$
\mathrm{H}_{0} \text { : discrepância } \geq 0,256 \mathrm{~m} \text { e } \mathrm{H}_{1} \text { : discrepância }<0,256 \mathrm{~m}
$$

O teste de sequências para a mediana das discrepâncias EN do ortomosaico Canon ELPH 110S foi realizado da seguinte maneira, Tabela 4:

Tabela 4 - Teste de aleatoriedade para as discrepâncias EN do ortomosaico obtido através da câmera Canon ELPH 110S, com base nas hipóteses previas onde Ho $\geq 0,256$ m e H1 < 0,256 $\mathrm{m}$.

\begin{tabular}{cccccccc}
\hline $\mathrm{m}$. & & & & & & & \\
\hline 0,323 & 0,291 & 0,245 & 0,212 & 0,684 & 1,080 & 1,065 & 0,789 \\
$\mathrm{~S}$ & $\mathrm{~S}$ & $\mathrm{~N}$ & $\mathrm{~N}$ & $\mathrm{~S}$ & $\mathrm{~S}$ & $\mathrm{~S}$ & $\mathrm{~S}$ \\
\hline 0,171 & 0,580 & 0,157 & 0,267 & 0,192 & 0,143 & 0,139 & 0,069 \\
$\mathrm{~N}$ & $\mathrm{~S}$ & $\mathrm{~N}$ & $\mathrm{~S}$ & $\mathrm{~N}$ & $\mathrm{~N}$ & $\mathrm{~N}$ & $\mathrm{~N}$ \\
\hline 0,171 & 0,238 & 0,644 & 0,155 & 0,151 & 0,201 & 0,143 & 0,165 \\
$\mathrm{~N}$ & $\mathrm{~N}$ & $\mathrm{~S}$ & $\mathrm{~N}$ & $\mathrm{~N}$ & $\mathrm{~N}$ & $\mathrm{~N}$ & $\mathrm{~N}$ \\
\hline 1,445 & 0,296 & 0,528 & 0,288 & & & & \\
$\mathrm{~S}$ & $\mathrm{~S}$ & $\mathrm{~S}$ & $\mathrm{~S}$ & & & & \\
\hline
\end{tabular}

Fonte: Elaborada pelos autores. 
As discrepâncias EN foram ordenadas e receberam a letra $\mathrm{S}$ (sim) quando a favor de $\mathrm{H}_{0}$ e $\mathrm{N}$ (não), quando contra $\mathrm{H}_{0}$. Na Tabela 5 é apresentada a realização do teste de sequências.

Tabela 5 - Teste de aleatoriedade das discrepâncias E N verificadas no ortomosaico Sequoia

\begin{tabular}{cccccccc}
\hline 0,405 & 0,216 & 0,339 & 0,464 & 0,470 & 0,809 & 0,811 & 0,209 \\
$\mathrm{~S}$ & $\mathrm{~N}$ & $\mathrm{~S}$ & $\mathrm{~S}$ & $\mathrm{~S}$ & $\mathrm{~S}$ & $\mathrm{~S}$ & $\mathrm{~N}$ \\
\hline 0,186 & 0,117 & 0,239 & 0,167 & 0,152 & 0,187 & 0,414 & 0,264 \\
$\mathrm{~N}$ & $\mathrm{~N}$ & $\mathrm{~N}$ & $\mathrm{~N}$ & $\mathrm{~N}$ & $\mathrm{~N}$ & $\mathrm{~S}$ & $\mathrm{~S}$ \\
\hline 0,252 & 0,248 & 0,190 & 0,278 & 0,326 & 0,201 & 0,202 & 0,158 \\
$\mathrm{~N}$ & $\mathrm{~N}$ & $\mathrm{~N}$ & $\mathrm{~S}$ & $\mathrm{~S}$ & $\mathrm{~N}$ & $\mathrm{~N}$ & $\mathrm{~N}$ \\
\hline 0,426 & 0,559 & 0,514 & 0,427 & & & & \\
$\mathrm{~S}$ & $\mathrm{~S}$ & $\mathrm{~S}$ & $\mathrm{~S}$ & & & & \\
\hline
\end{tabular}

Fonte: Elaborada pelos autores.

De acordo com a sequência acima, tem-se que $\mathrm{R}=9$, para $\mathrm{n}_{1}=14$ e $\mathrm{n}_{2}$ $=14$. Como $\mathrm{n}_{1}$ e $\mathrm{n}_{2}$ são menores que 15 , os valores limites de $\mathrm{R}$ foram extraídos da tabela de valores críticos para o teste de sequência, onde se obteve $c_{1}$ igual a 9 e $c_{2}$ igual a 21 . Como $R$ é igual a 9 , não se rejeita a hipótese nula ao nível de 95\% de confiança. Assim, as discrepâncias E N foram consideradas aleatoriamente distribuídas.

3.3 Análise de erros sistemáticos por meio da estatística circular

As discrepâncias $\mathrm{E} \mathrm{N}$, os azimutes, e o quadrante de $\theta$ são exemplificados nas Tabelas 6 e 7. E, na Tabela 8 são apresentados os resultados da análise direcional de acordo com as Eq. 14 a 19. Na Figura 6, a representação no círculo de azimutes, dos vetores do erro direcional, bem como a resultante. E, na Tabela 9, os resultados dos testes de significância e uniformidade para as direções analisadas nos dois ortomosaicos, Canon ELPH 110 S e Sequoia. 
Tabela 6 - Exemplo de azimutes das discrepâncias e E N observados no ortomosaico gerado com imagens da câmera Canon ELPH 110 S.

\begin{tabular}{ccccc}
\hline $\begin{array}{c}\text { Ponto de } \\
\text { Checagem }\end{array}$ & Disc. E (m) & Disc N (m) & $\begin{array}{c}\text { Azimute } \\
\text { (graus dec) }\end{array}$ & Quadrante \\
\hline 1 & 0,098 & $-0,308$ & 162,3369295 & $\mathrm{~S}$ \\
2 & 0,178 & $-0,230$ & 142,2803561 & $\mathrm{SE}$ \\
3 & 0,239 & $-0,054$ & 102,8456149 & $\mathrm{E}$ \\
$\ldots$. & $\ldots \ldots$ & $\ldots \ldots$ & $\ldots \ldots$ & $\ldots$. \\
13 & 0,153 & 0,115 & 53,0900225 & $\mathrm{NE}$ \\
14 & $-0,115$ & $-0,085$ & 233,4313672 & $\mathrm{SW}$ \\
15 & 0,006 & 0,139 & 2,4478482 & $\mathrm{~N}$ \\
16 & 0,045 & $-0,053$ & 139,3254005 & $\mathrm{SE}$ \\
$\ldots$. & $\ldots$. & $\ldots$. & $\ldots$. & $\ldots$. \\
29 & 0,264 & $-0,133$ & 116,7823105 & $\mathrm{SE}$ \\
30 & 0,445 & $-0,284$ & 122,5728078 & $\mathrm{SE}$ \\
31 & 0,263 & $-0,116$ & 113,8107428 & $\mathrm{SE}$ \\
\hline
\end{tabular}

Fonte: Elaborada pelos autores.

Tabela 7 - Exemplo de azimutes das discrepâncias EN observados no ortomosaico gerado com imagens da câmera Sequoia

\begin{tabular}{ccccc}
\hline $\begin{array}{c}\text { Ponto de } \\
\text { Checagem }\end{array}$ & Disc. E (m) & Disc N (m) & $\begin{array}{c}\text { Azimute } \\
\text { (graus dec) }\end{array}$ & Quadrante \\
\hline 1 & 0,390 & 0,390 & 105,4786382 & $\mathrm{E}$ \\
2 & $-0,076$ & $-0,076$ & 200,6181882 & $\mathrm{~S}$ \\
3 & 0,146 & 0,146 & 154,4930619 & $\mathrm{SE}$ \\
$\ldots$ & $\ldots$. & $\ldots$. & $\ldots$. & $\ldots$. \\
13 & 0,005 & 0,005 & 1,884050214 & $\mathrm{~N}$ \\
14 & $-0,011$ & $-0,011$ & 356,6335393 & $\mathrm{~N}$ \\
15 & 0,012 & 0,012 & 1,660282368 & $\mathrm{~N}$ \\
16 & $-0,003$ & $-0,003$ & 359,3489396 & $\mathrm{~N}$ \\
$\ldots$ & $\ldots$. & $\ldots$. & $\ldots$. & $\ldots$. \\
29 & 0,508 & 0,508 & 114,6390974 & $\mathrm{SE}$ \\
30 & 0,493 & 0,493 & 106,4964471 & $\mathrm{E}$ \\
31 & 0,401 & 0,401 & 110,0060477 & $\mathrm{E}$ \\
\hline
\end{tabular}

Fonte: Elaborada pelos autores.

Tabela 8 - Resultantes, média direcional e variância das discrepâncias observadas nos ortomosaicos provenientes das imagens dos sensores Canon e Sequoia.

\begin{tabular}{cccccc}
\hline Ortomosaico & $\bar{S}(\mathrm{~m})$ & $\bar{C}(\mathrm{~m})$ & $\bar{R}(\mathrm{~m})$ & $\theta$ (graus) & Variância \\
\hline $\begin{array}{c}\text { Canon ELPH } \\
\text { 110S }\end{array}$ & 0,035 & 0,114 & 0,192 & 47 & 0,880 \\
Sequóia & 0,124 & 0,146 & 0,189 & 116 & 0,810 \\
\hline
\end{tabular}

Fonte: Elaborada pelos autores.

Como pode ser observado na Tabela 8, a variância das direções das discrepâncias direcionais observadas no ortomosaico gerado com imagens dos sensores Canon ELPH 110 S, foi de 0,880 e a variância do ortomosaico gerado 
com imagens do sensor Sequoia foi 0,810. Como discutido por Santos et al. (2016), como a variância é próxima ao valor 1, indica que não há tendência nos dois ortomosaicos, como mostrado na Figura 7, onde nota-se que as direções das discrepâncias estão distribuídas por todo o círculo de azimutes. Uma translação, no sentido contrário das médias direcionais, 470 e $116^{\circ}$ poderia ser aplicada nos ortomosaicos, caso o resultado da variância indicasse pela tendência.

Figura 7 - Distribuição dos vetores direcionais e resultante das discrepâncias EN dos ortomosaicos Canon ELPH 110S (a) e Sequoia (b), com base na função de distribuição de von Mises, $\mathrm{k}=0,5$.

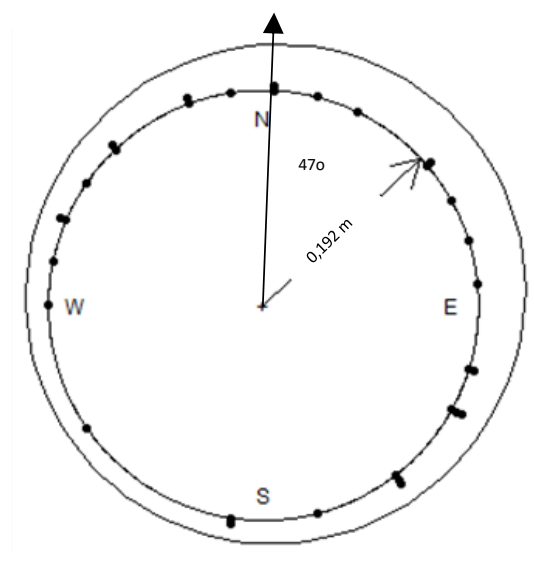

(a)

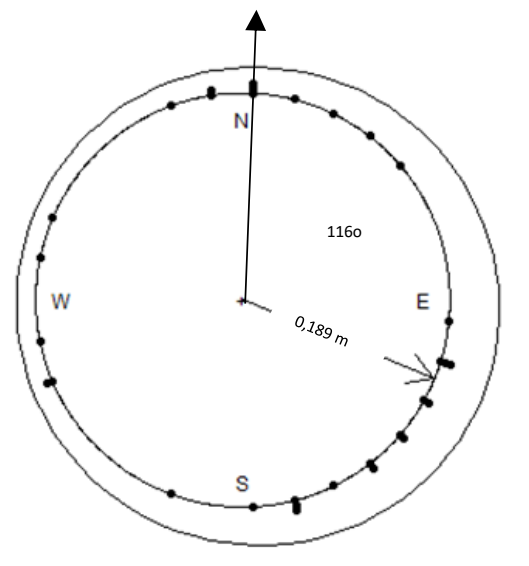

(b)

Fonte: Elaborada pelos autores.

Tabela 9 - Resultados do teste de significância e uniformidade para as direções dos escorregamentos das discrepâncias $\mathrm{E}$ e $\mathrm{N}$ dos ortomosaicos provenientes das imagens dos sensores Canon ELPH 110 S e Sequoia

\begin{tabular}{ccccccc} 
Ortomosaico & \multicolumn{2}{c}{$\begin{array}{c}\text { Teste de Rayleigh } \\
\text { (média) }\end{array}$} & \multicolumn{2}{c}{$\begin{array}{c}\text { Teste de } \\
\text { Uniformidade }\end{array}$} & \multicolumn{2}{c}{$\begin{array}{c}\text { Teste de } \\
\text { Aleatoriedade }\end{array}$} \\
& Zcalc & Zcrít & $\chi^{2}$ calc & $\chi^{2}$ crít & Zcalc & $\mathrm{P}($ Zcalc) \\
& & $(30,0,05)$ & & $(30,0,05)$ & & \\
\hline $\begin{array}{c}\text { CANON } \\
\text { ELPH 110 S }\end{array}$ & 0,444 & 2,971 & 4,355 & 43,773 & 0,001 & 0,500 \\
Sequoia & 1,136 & 2,971 & 12,161 & 43,773 & 0,002 & 0,500
\end{tabular}

Fonte: Elaborada pelos autores.

Rev. Bras. de Cartografia, vol. 70, Edição Especial "XXVII Congresso Brasileiro de 
O teste de Rayleigh demonstrou que existe uma média direcional para os azimutes das discrepâncias horizontais dos ortomosaicos, oriundos do processamento das imagens CANON ELPH 110 S e Sequoia. Nos dois casos, Zcal foi menor que Zcrit de acordo com a tabela de valores limites deste teste $(0,444<2,971$ e $1,136<2,971)$. Assim, aceita-se a hipótese nula ao nível de significância de 0,05 e com 30 graus de liberdade.

Os valores de qui-quadrado calculados (4,355 para Canon ELPH 110S e 12,161 para Sequoia) para as discrepâncias direcionais no teste de uniformidade foram menores que qui-quadrado crítico $(43,773)$ nos dois casos, conduziram a não rejeição da hipótese nula com 30 graus de liberdade e a nível de confiança de $95 \%$. Ou seja, os azimutes das discrepâncias apresentam distribuição uniforme em ambos ortomosaicos.

No teste de aleatoriedade, o valor de Zcal apresentou probabilidade igual a 1 para a distribuição espacial dos azimutes. Desta maneira, não se rejeita a hipótese nula ao nível de confiança de 95\% para os ortomosaicos obtidos através dos sensores Canon ELPH 110S e Sequóia.

\subsection{Análise da exatidão posicional}

A exatidão posicional dos ortomosaicos foram avaliadas por meio do RMSE, Equação 24, confrontando-se com o PEC-PCD para determinar a classe e a escala de enquadramento, como apresentado na Tabela 10.

Tabela 10 - Resultados da análise de exatidão horizontal dos ortomosaicos provenientes das imagens dos sensores Canon e Sequoia com referência no PEC-PCD.

\begin{tabular}{ccccc}
\hline Ortomosaico & RMSE (m) & PEC-PCD (m) & Classe & Escala \\
\hline $\begin{array}{c}\text { Canon ELPH } \\
\text { 110S }\end{array}$ & 0,514 & 0,60 & B & $1: 2000$ \\
Sequoia & 0,375 & 0,60 & B & $1: 2000$ \\
\hline
\end{tabular}

Fonte: Elaborada pelos autores. 
Desta forma, os ortomosaicos gerados com imagens coletadas com os sensores da câmera Canon ELPH $110 \mathrm{~S}$ e sequoia processados pela técnica SfM enquadraram-se na escala 1:2000, classe B, de acordo com o PEC-PCD.

\section{Conclusão}

Conclui-se com este trabalho que a avaliação prévia da função de distribuição da amostra quanto a normalidade pode conduzir a adoção de métodos não paramétricos para análise da exatidão de produtos cartográficos.

A estatística direcional permite conhecer a existência de erros sistemáticos ou não, por meio da variância direcional e da distribuição dos vetores de discrepâncias no círculo azimutal é bastante simples.

A exatidão posicional dos ortomosaicos gerados com as imagens dos sensores Canon ELPH 110S e sequoia analisados por meio das discrepâncias entre pontos homólogos apresentaram distribuição não normal da função de distribuição de probabilidades e distribuição aleatória.

$\mathrm{O}$ teste direcional demonstrou que ambos ortomosaicos não apresentaram erros sistemáticos. Uma constatação é que os vetores das discrepâncias apresentaram direções diferentes no círculo azimutal, embora as imagens tenham sido coletadas sob as mesmas condições de vôo, processadas pelo mesmo método, com os mesmos pontos de controle, e ainda, checados por meio dos mesmos pontos de checagem. Assim, pode-se atribuir que as diferenças na direção do vetor resultantes podem ter sido influenciadas pelas particularidades de cada sensor utilizado.

E, pela análise do RMSE confrontando com o PEC-PCD, estes enquadraram-se na Classe B da escala 1:2000.

\section{Agradecimentos}


À Fundação para o Incremento da Pesquisa e Aperfeiçoamento Industrial - FIPAI de São Carlos, SP.

Ao Grupo de Trabalho do Laboratório de Visualização Avançada da Universidade do Vale do Rio dos Sinos.

\section{Referências}

AMERICAN SOCIETY PHOTOGRAMMETRY AND REMOTE SENSING ASPRS. Positional Accuracy Standards for Digital Geospatial Data. Photogrammetric Engineering \& Remote Sensing, v. 81, No. 3, 2015. pp. A1-A26.

ARIZA, F. J. L; ATKINSON, A. D. G. Analysis of Some Positional Accuracy Assessment Methodologies. Journal of Surveying Engineering, Vol. 134, No. 2. 2008. pp. 45-54.

ATKInson GORDO, A. D. J, 2005. Control de Calidad Posicional en Cartografía: Análisis de los principales estándares y propuesta de mejora. Universidad de Jaén, Jaén - Espanha. 370 páginas.

BRASIL, 1984. Decreto no. 89.817 de 20 de Junho de 1984. Normas Técnicas da Cartografia Nacional. Brasil. http://www.planalto.gov.br/ccivil_03/decreto/1980-1989/D89817.htm.

BROWN, M.; LOWE, D. Invariant Features from Interest Point Groups. In: Proceedings of the, Anais... In: BRITISH MACHINE CONFERENCE BMVC. 2002.

CORSI, C.; SLAPŠAK, B.; VERMEULEN, F. Good Practice in Archaeological Diagnostics: Non-invasive Survey of Complex Archaeological Sites. [s.l.] Springer Science \& Business Media, 2013. COVENEY, S.; ROBERTS, K. Lightweight UAV digital elevation models and orthoimagery for environmental applications: data accuracy evaluation and potential for river flood risk modelling. International Journal of Remote Sensing, Vol. 38, No. 8-10. 2017. pp. 3159-3180. 
DEBEVEC, P. E. et al. Modeling and Rendering Architecture from Photographs: A hybrid geometry- and image-based approach. SIGGRAPH conference proceedings. $1996.10 \mathrm{p}$.

DOMINGO, A. M. Aplicación de las Técnicas de Estimación Robusta en algunos problemas fotogramétricos: I: Detección de Errores en el Proceso de Orientación Relativa Analítica. Universidad Politécnica de Madrid, 2000 .

FISHER, N. I. Statistical Analysis Of Circular Data. UK: Cambridge University Press, 1993. 277 páginas.

FONSTAD, M. A. et al., Topographic Structure from Motion: A New Development in Photogrammetric Measurement. Earth Surface Processes and Landforms, Vol. 38, No. 4, 2013. pp. 421-430.

GARCÍA, J. L. L. Fotogrametría moderna: analítica y digital. Universidad Politécnica de Valencia, Espanha. 2002. 564 páginas.

HARTLEY, R.; ZISSERMAN, A. Multiple View Geometry in Computer Vision. 2. ed. [s.l.] Cambridge, 2003. v. 1

HARWIN, S.; LUCIER, A. Assessing the Accuracy of Georeferenced Point Clouds Produced via Multi-View Stereopsis from Unmanned Aerial Vehicle (UAV) Imagery. Remote Sensing, Vol. 4, No. 12, 30. 2012. pp. 1573-1599.

JAMMALAMADAKA, S. R.; SEN-GUPTA, A. Topics in Circular Statistics. River Edge, N. J.: World Scientific. Vol. 5. 2001. 336 pag.

JAUD, M. et al. Assessing the Accuracy of High Resolution Digital Surface Models Computed by PhotoScan ${ }^{\circledR}$ and MicMac ${ }^{\circledR}$ in Sub-Optimal Survey Conditions. Remote Sensing, Vol. 8, No. 6, 2016. pp. 1-18.

JEONG, Y. et al. Pushing the Envelope of Modern Methods for Bundle Adjustment. IEEE transactions on pattern analysis and machine intelligence, v. 34, n. 8, 2012, pp. 1605-1617.

JUPP, P. E e MARDIA, K. V. A Unified View of the Theory of Directional Statistics, 1975-1988. International Statistical Review / Revue Internationale de Statistique. Vol 57, No. 3. 1989. pp. 261-294. 
LOWE, D. G. Object Recognition from Local Scale-Invariant Features 1. Introduction. In: Proc., Corfu, Grecia. Anais... In: INTERNATIONAL CONFERENCE ON COMPUTER VISION. Corfu, Grecia: 1999.

MANN, P. S. Introdução à Estatística. Tradução: Eduardo Benedito Curtulo, Teresa Cristina Padilha de Souza. 5. ed. Rio de Janeiro: LTC, 2006.

MERCHANT, D. C. Spatial Accuracy for Large Scale Line Maps. In Technical Congress of Surveing and Mapping. 1982. pp. 222-231.

MOREELS, P.; PERONA, P. Evaluation of Features Detectors and Descriptors Based on 3D Objects. International Journal of Computer Vision, v. 73, n. 3, 2007, pp. 263-284.

OTIENO, B. S, 2002. An alternative estimate of preferred direction for circular data. Virginia Polytechnic Institute and State University, Blacksburg, Virginia, 219 páginas.

REMONDINO, F. et al. UAV photogrammetry for mapping and $3 \mathrm{~d}$ modeling - current status and future perspectives. In Anais Photogrammetry, Remote Sensing and Spatial Information Sciences. Zurich, Suiça: ISPRS, 2011. pp. 25-31.

SANTOS, A. P. Controle de qualidade cartográfica: metodologias para avaliação da exatidão posicional em dados espaciais. Universidade Federal de Viçosa, Viçosa - MG, 2015.172 páginas.

SANTOS, A. P. et al. Avaliação da Acurácia Posicional em Dados Espaciais Utilizando Técnicas de Estatística Espacial: Proposta de Método e Exemplo Utilizando a Norma Brasileira. Boletim de Ciências Geodésicas. Vol. 22, nº. 4. Curitiba - PR. Out/ dez, 2016. pp 630-650.

SNAVELY, N. et al. R. Modeling the World from Internet Photo Collections. International Journal of Computer Vision, Vol. 80, No. 2, nov. 2008. pp. 189-210.

SNAVELY, N.; SEITZ, S. M.; SZELISKI, R. Modeling the World from Internet Photo Collections. International Journal of Computer Vision, v. 80, n. 2, 2008, pp. 189-210. 
SZELISKI, R., 2010. Computer vision: algorithms and applications. Washington dc, EUA: Springer. Vol. 1. 979 páginas.

TORMAN, V. B. L.; COSTER, R.; RIBOLDI, J. Normalidade de variáveis: métodos de verificação e comparação de alguns testes não-paramétricos por simulação Normalidade. Rev. HCPA, v. 32, n. 2, 2012, pp. 227-234. TRIGGS, B. et al. Bundle Adjustment - A Modern Synthesis - Vision Algorithms: Theory \& Practice. [s.1.] A. Zisserman \& R. Szeliski (Eds.), Springer-Verlag LNCS 1883, 2000. 